EBERHARD, ABENDLANDISCHE ERZIEHUUNGSWEISHEIT 



\title{
ABENDLANDISCHE ERZIEHUNGSWEISHEIT
}

EINE HILFE FƯR DIE NOT DER GEGENWART

\author{
VON \\ OTTO EBERHARD
}

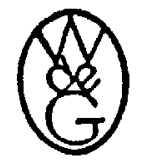

WALTER DE GRUYTER \& CO.

VORMALS G. J. GOSCHEN'SCHE VERLAGSHANDLUNG / J. GUT'TENTAG, VERLAGSBUCHHANDLUNG / GEORG REIMER / KARL J. TRUBNER / VEIT \& COMP. 
(C)

Copgright 1958 by Walter de Gruyter \& Co., Berlin W 35

Printed in Germany

Archiv-Nr. 426056

Alle Rechte, einschließlich des Rechtes der Herstellung ron Fotoloplen und Mikrofilmen, vorbehalten

Satz und Druck: Berliner Buchdruckerei Union GmbH., Berlia SW 61 


\author{
MEINER ALTERSWIRKSTATTE \\ DER GEORG-HERWEGH-SCHULE, GYMNASIUM, \\ IN BERLIN-HERMSDORF \\ UND IHRER ELTERNSCHAFT ZUM GRUSSE
}


